$$
\mathrm{CO}_{2}+\mathrm{SO}_{2}+\mathrm{CuO}=\mathrm{CuSO}_{4}+\mathrm{CO}
$$

Reactions (C-1) and (17) have been established and Reaction (18) is thermodynamically favorable. In this cycle, other metals, such as manganese and magnesium, may be substituted for copper.

In consequence, one of the most promising cycles may be a sulfate-based cycle consisting of Reactions (C-1), (17) and (18). This cycle has the following features:

(i) It uses only carbon dioxide and heat energy at temperatures below $1000 \mathrm{~K}$.

(ii) The change in the standard Gibbs free energy for each constituent reaction is less than $40 \mathrm{~kJ} / \mathrm{mol}$.

(iii) As it consists of both endothermic and exothermic reactions, a high thermal efficiency is attainable.

(iv) The reactions in the cycle are for the most part gas-solid reactions. Therefore, the reaction products can easily be separated, but a technique for separating gaseous components at high temperatures will be required.

\section{Conclusions}

An evolutional method for synthesizing chemical reaction cycles is proposed in which chemical reactions are represented with a directed graph. A search for reaction sequence is carried out for the reduction of carbon dioxide to monoxide.

As a result, a tin-based cycle and a sulfate-cycle are selected and the latter can be a promising cycle.

\section{Acknowledgment}

The authors wish to express their thanks to the Science Research Foundation of the Ministry of Education, Science and Culture, Japan, for its financial support (Grant No. 57040043).

\section{Literature Cited}

1) Bamberger, C. E. and P. R. Robinson, Inorganica Chimica Acta, 42, 133 (1980).

2) Kameyama, H., K. Yoshida and D. Kunii, The Chem. Eng. J., 11, 223 (1976).

3) Martin, L. R., Solar Energy, 24, 271 (1980).

\title{
A SIMULATION OF CONTINUOUS SUSPENSION POLYMERIZATION OF STYRENE BY THE MONTE CARLO METHOD
}

\author{
YASUO HATATE AND ATSUSHI IKARI \\ Department of Chemical Engineering, Kagoshima University, Kagoshima 890 \\ FUMIYUKI NAKASHIO AND KAZUO KONDO \\ Department of Organic Synthesis, Kyushu University, Fukuoka 812
}

Key Words: Chemical Reaction, Styrene Suspension Polymerization, Coalescence, Monte Carlo Method,

Polymer Chain Length

\section{Introduction}

In the previous paper, ${ }^{2)}$ the effect of the interaction between droplets on monomer conversion in continuous suspension polymerization was estimated by the Monte Carlo method. The chain length of polymer is also important in specifying the reactor performance as well as the monomer conversion. In this paper, the chain length of the polymer produced is calculated by the Monte Carlo method, and the

Received June 20,1983. Correspondence concerning this article should be addressed to Y. Hatate. effect of the interaction between polymer droplets on chain length is discussed.

\section{Kinetics of Polymerization Reaction ${ }^{4-5,7)}$}

In a free-radical polymerization involving initiation, propagation, transfer to monomer and termination by combination, the reaction rates of monomer ( $=\mathrm{A})$ and dead polymer of chain length $n$ $\left(=M_{n}\right)$ are expressed as follows:

$$
R_{p}=-\rho \frac{d(A / \rho)}{d t}=\frac{\gamma}{\delta_{A}{ }^{\prime}} A I^{1 / 2}
$$




$$
\begin{aligned}
R_{M_{n}} & =\rho \frac{d\left(M_{n} / \rho\right)}{d t} \\
& =(1-\zeta) \zeta^{n-1} \frac{R_{i}{ }^{1 / 2}}{\delta_{A} \nu}\left\{T_{f A} A+\frac{(n-1)(1-\zeta)}{2 \zeta} \delta_{A} \nu R_{i}{ }^{1 / 2}\right\}
\end{aligned}
$$

where $R_{i}$ is the rate of initiation and $\zeta$ is a probability factor. They are written as

$$
\begin{aligned}
& R_{i}=2 k_{d} f \in I \\
& \zeta=1-\left(T_{f A}+R_{i} / R_{p}\right)
\end{aligned}
$$

In these equations, $\gamma$ and $\varepsilon$, respectively, are the correction factors for the overall rate constant of monomer consumption and for the initiator efficiency in the gel effect region, which are shown as functions of polymer weight fraction in the polymer droplets. In the range of initiator $\left(2,2^{\prime}\right.$-azobisisobutyronitrile) concentration $0.03-0.09 \mathrm{~mol} / \mathrm{dm}^{3}$, these correlations are applicable in describing the monomer conversion and the average degree of polymerization $(\overline{D P})$ up to almost the complete conversion. ${ }^{2-5,7,9)}$ The number and weight average chain length are calculated from the following equations:

$$
\begin{aligned}
& \overline{D P}_{N}=\Sigma n M_{n} / \Sigma M_{n} \\
& \overline{D P} \bar{P}_{W}=\Sigma n^{2} M_{n} / \Sigma n M_{n}
\end{aligned}
$$

Assuming $\omega=\infty$ in CSTR, the following equation is obtained from mass balance. ${ }^{5)}$

$$
R_{p}{ }^{\prime}=\frac{\left(A_{f} / \rho_{f}\right)}{\theta} X_{A}
$$

where $R_{p}{ }^{\prime}\left(=R_{p} / \rho\right)$ is the monomer consumption rate per unit mass.

\section{Calculation by Monte Carlo Method}

As shown in the previous paper, ${ }^{2)}$ the following simplified assumptions are necessary to obtain a solution.

a) All the polymer droplets are uniform in mass and constant in number, in the stirred-tank reactor.

b) Two of the droplets coalesce at a time and redisperse instantly to form two identical droplets.

c) The probability of coalescing and redispersing is the same for all droplets.

d) The concentration is uniform within each droplet.

e) The continuous phase is homogeneous and the reaction only takes place in the droplets.

The calculation based on the Monte Carlo method was carried out by the following procedure. First, the initial condition for each droplet $(k=1,2, \cdots N)$ is set up, where $k$ is the assignment number of droplet and $N$ is the total number of droplets. Iteration number $l$ is set to be unity.
1) Random numbers $R_{j}(j=1,2, \cdots N / \theta \cdot \Delta T)$ are generated from the uniform random numbers ranging from 1 to $N$. $N$ or $\Delta T$ should be adjusted to make an integer value of $N / \theta \cdot \Delta T$. The droplet having assignment number $R_{j}$ is discharged from the reactor, and the inlet condition is set up for the droplet of $k=R_{j}$.

2) I sets of a pair of random numbers are generated. The droplets having assignment numbers $R_{2 I-1}$ and $R_{2 I}$ coalesce and redisperse. They are averaged to have the same concentration.

3) Batch reaction is carried out within each droplet for the time $\Delta T(=\theta / N)$. The concentrations of $A$ and $M_{n}$ after the reaction time $\Delta T$ are calculated using the Runge-Kutta-Gill method as follows:

$$
\begin{gathered}
\left.\left.\frac{A}{\rho}\right]_{l+1}=\frac{A}{\rho}\right]_{l}+\int_{0}^{\Delta T} R_{p} d t \\
\left.\left.\frac{M_{n}}{\rho}\right]_{l+1}=\frac{M_{n}}{\rho}\right]_{l}+\int_{0}^{\Delta T} R_{M_{n}} d t \\
l=l+1
\end{gathered}
$$

$R_{p}$ and $R_{M_{n}}$ are given in Eqs. (1) and (2), respectively. The concentration $\bar{M}_{n}$ at elapsed time $t(=l \cdot \Delta T)$ is obtained from averaging $M_{n}$ of each droplet by the following equation.

$$
\left(\bar{M}_{n} / \rho\right)=\sum_{k=1}^{N}\left(M_{n} / \rho\right)_{k} / N
$$

The procedure 1) to 3 ) is iterated until steady state is reached. In most cases it is realized at $t \leqq 5 \cdot \theta$. Coalescence and redispersion rate $\omega$ is expressed by $2 I / \theta$.

Three different conditions such as zero conversion, $100 \%$ conversion and steady state under complete segregation* are adopted as initial conditions for 1000 droplets, and the steady state values of monomer coversion and $\overline{D P}$ at a very long process time, that is, 5 times the mean residence time $(\theta)$ or more, are determined for each case. The probable value of $\omega$ can be estimated from the physical properties of liquids, reactor design and operating conditions. ${ }^{6,9)}$

\section{Results and Discussion}

Two levels, 0.06 and $0.03 \mathrm{~mol} / \mathrm{dm}^{3}$, have been selected as initiator concentration, because 0.06 $\mathrm{mol} / \mathrm{dm}^{3}$ was mainly used in the previous experimental work ${ }^{3)}$ and $0.03 \mathrm{~mol} / \mathrm{dm}^{3}$ seems to be a more general condition in the free radical polymerization using AIBN as initiator.

Since the effect of coalescence and redispersion on conversion was described in the previous paper, ${ }^{2)} \overline{D P}$ is discussed here.

In regard to the transient behavior of $\overline{D P}$ at various conditions, the same aspects as shown in the case of

\footnotetext{
* No interaction between droplets.
} 
conversion have been recognized as follows:

1) Under a certain small interaction between droplets such as $\omega=0.2^{*}$, an abrupt increase of $\overline{D P}$ with increase of process time may be found in the plot of $\overline{D P}$ vs. process time with $\theta$ as parameter, where the steady state with no interaction between droplets $(\omega=0)$ is selected as the initial condition.

2) In a certain range of $\theta, \overline{D P}$ might converge to different steady state values at a long process time, depending on their initial conditions. This is due to the multiple steady states peculiar to CSTR having an auto-acceleration effect. ${ }^{1)}$ To simplify the explanation of this phenomenon, the condition of $\omega=\infty$ is selected, and the possibility of the existence of multiple steady states is shown graphically. Equation (7) is transformed into the following equation.

$$
\begin{aligned}
R_{p 0}^{\prime} & \equiv \frac{\gamma}{\delta_{A}{ }^{\prime}}\left(\frac{A_{f}}{\rho_{f}}\right)\left(1-X_{A}\right) I_{f}{ }^{1 / 2}=\frac{\left(1+k_{d} \theta\right)^{1 / 2}}{\theta}\left(\frac{A_{f}}{\rho_{f}}\right) X_{A} \\
& \equiv G(\theta)
\end{aligned}
$$

Using the above equation, the value $X_{A}$ vs. an arbitrary $\theta$ is determined from the intersection of $R_{p 0}^{\prime}$ vs. $X_{A}$ curve and $G(\theta)$ and $X_{A}$ line as shown in Fig. 1. Calculated results of $R_{p 0}^{\prime}$ for $I_{f}=0.06 \mathrm{~mol} / \mathrm{dm}^{3}$ and $G(\theta)$ for $\theta=50,100,150,200$ and $300 \mathrm{~min}$ are shown against $X_{A}$ in the figure. Three intersection points are found in the cases of $\theta=150$ and $200 \mathrm{~min}$. The middle point among them for each case is so unstable that two stable steady states are confirmed. It is obvious that multiple steady states are obtained in the shadowed portion bounded by two dotted lines in the figure. It seems impossible to specify the condition of multiple steady states for finite values $\omega$ without the Monte Carlo method.

Steady values of calculated $\overline{D P}_{N}$ and $\overline{D P}_{W}$ are plotted against $\theta$ with $\omega$ as parameter under the condition of $I=0.06 \mathrm{~mol} / \mathrm{dm}^{3}$ in Figs. 2 and 3, respectively. The calculated results at $\omega \geq 2$ are the same as that at infinite $\omega$. The continuous suspension polymerization of $\omega=\infty$ is substantially the same as the continuous bulk polymerization in complete micro-mixing, where the auto-accelerating effect in the latter stage of the polymerization would be predominant, even with mechanical mixing. ${ }^{5)}$ The multiple steady states exist in the range shown by the dotted vertical lines in the figures. These regions, bounded by dotted and solid lines, become wide with increasing $\omega$.

Calculations of $\overline{D P}_{N}$ and $\overline{D P}_{W}$ vs. $\theta$ in the case of $I=0.03 \mathrm{~mol} / \mathrm{dm}^{3}$ are shown in Figs. 4 and 5 , respectively. As well as in the calculation of monomer conversion in the previous paper, ${ }^{2)}$ the range of

\footnotetext{
* One coalescence and redispersion per 5 minutes for each droplet.
}

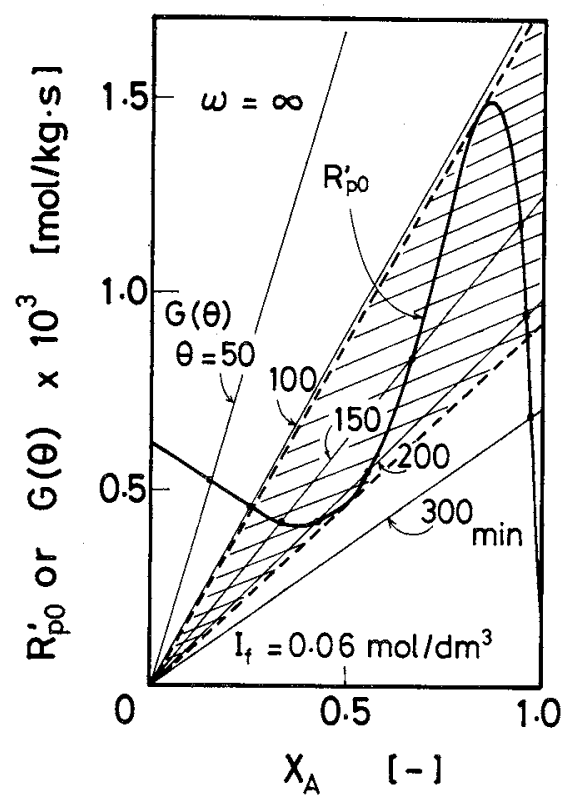

Fig. 1. Graphical verification of multiple steady states.

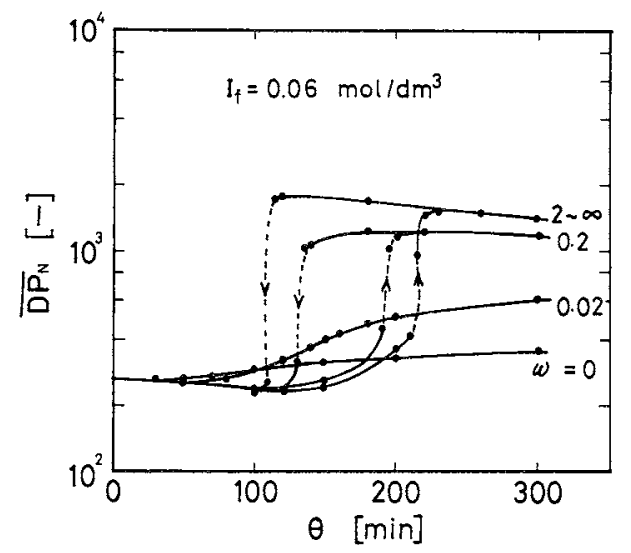

Fig. 2. Relations between number average chain length and mean residence time for $I_{f}=0.06 \mathrm{~mol} / \mathrm{dm}^{3}$.

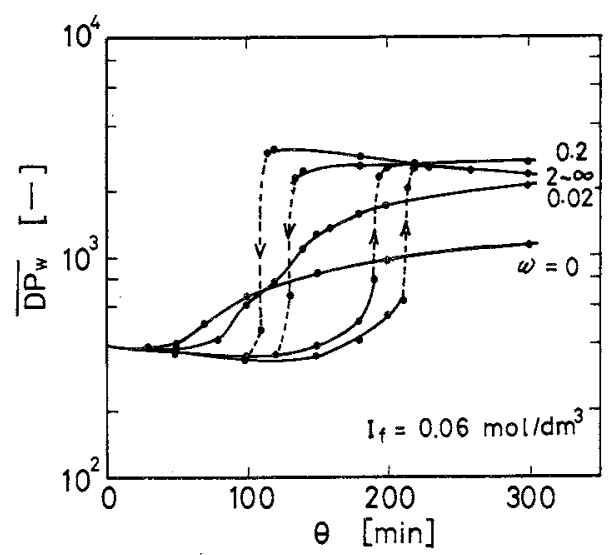

Fig. 3. Relations between weight average chain length and mean residence time for $I_{f}=0.06 \mathrm{~mol} / \mathrm{dm}^{3}$.

multiple steady states of $\overline{D P}$ shifts to the direction of long mean residence time, compared with that of $I=$ $0.06 \mathrm{~mol} / \mathrm{dm}^{3}$. 


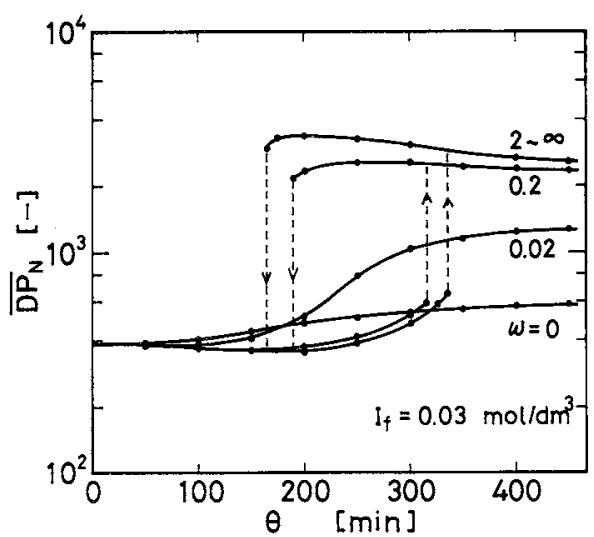

Fig. 4. Relations between number average chain length and mean residence time for $I_{f}=0.03 \mathrm{~mol} / \mathrm{dm}^{3}$.

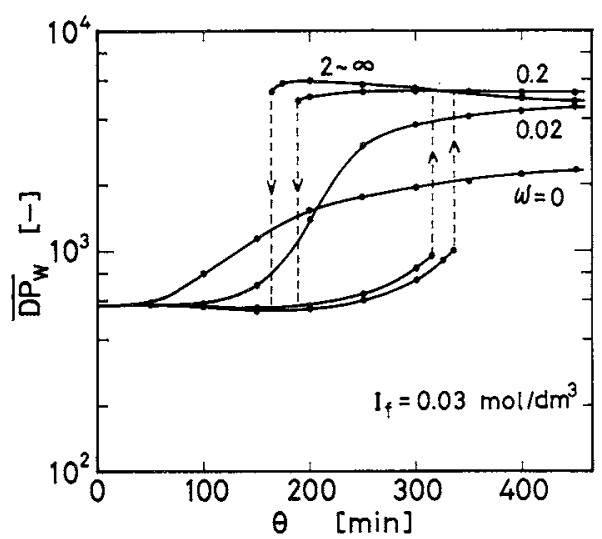

Fig. 5. Relations between weight average chain length and mean residence time for $I_{f}=0.03 \mathrm{~mol} / \mathrm{dm}^{3}$.

\section{Conclusion}

A simulation of continuous suspension polymerization of styrene was carried out by use of the Monte Carlo method.

The following conclusion has been obtained.

1. The effect of coalescence and redispersion on $\overline{D P}$ is found to be as strong as that on monomer conversion, which was discussed in the previous paper. $^{2)}$

2. Multiple steady states may be observed when a small interaction exists between droplets.

3. The assumption of complete micro-mixing ${ }^{5}$ ) would be applicable to the suspension polymerization of styrene having 2 or more interactions per minute for each polymer droplet.

\section{Nomenclature}

$A \quad=$ monomer or monomer concentration $\left[\mathrm{mol} / \mathrm{dm}^{3}\right]$

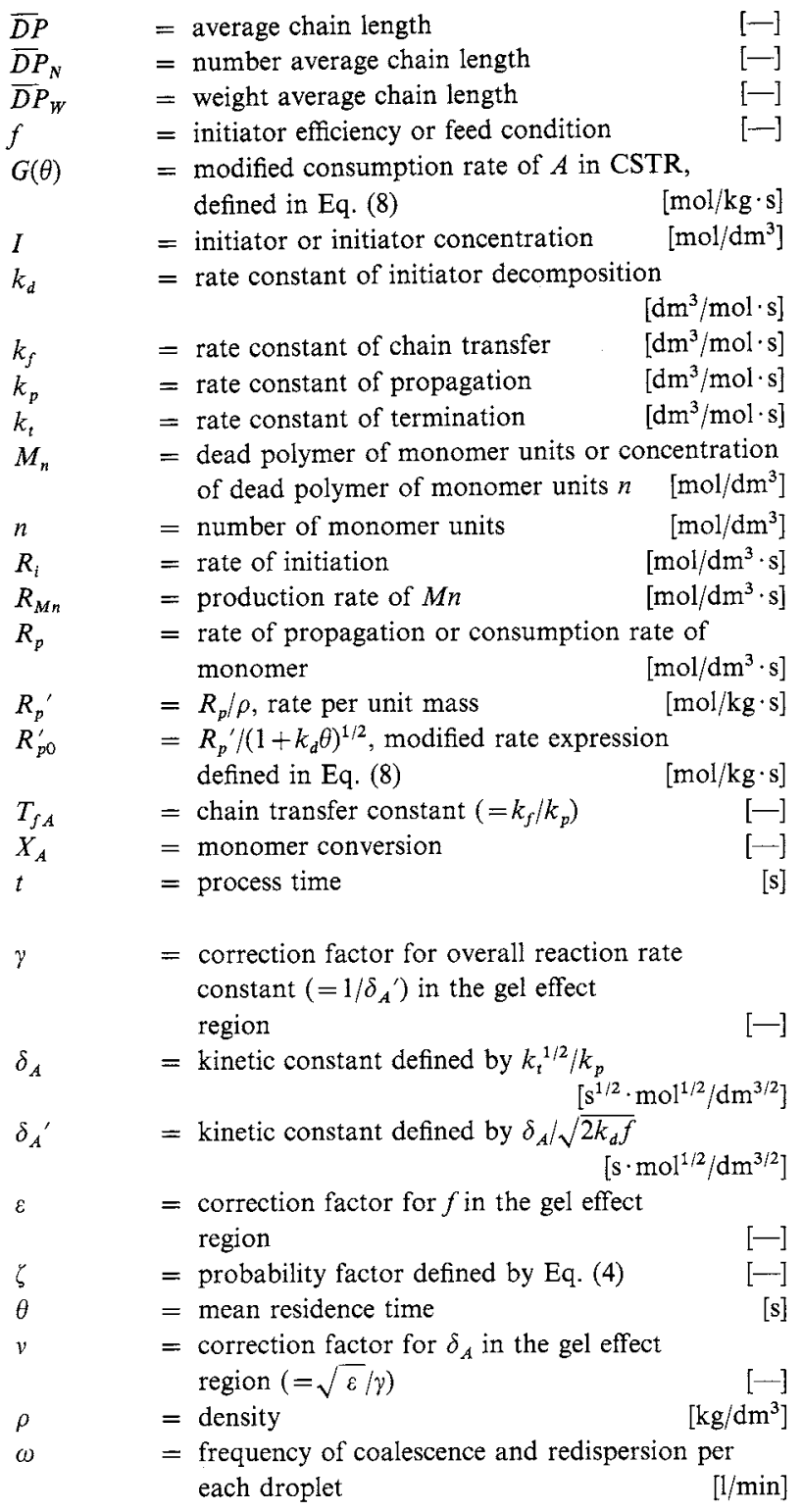

\section{Literature Cited}

1) Eguchi, W. and M. Harada: Kagaku Kögaku, 29, 605 (1965).

2) Hatate, Y., A. Ikari, F. Nakashio and K. Kondo: J. Chem. Eng. Japan, 14, 493 (1981).

3) Hatate, Y., T. Ikeura, M. Shinonome, K. Knodo and F. Nakashio: J. Chem. Eng. Japan, 14, 38 (1981).

4) Hatate, Y., F. Nakashio: Kagaku Kōgaku, 37, 171 (1973).

5) Hatate, Y., F. Nakashio and W. Sakai: J. Chem. Eng. Japan, 4, 348 (1971).

6) Komazawa, I.: "Kagaku Kikai Gijutsu," Vol. 22, The Inst. of Chem. Engrs., ed., pp. 1-24, Maruzen, Tokyo (1970).

7) Miyata, T. and F. Nakashio: Kagaku Kögaku, 37, 599 (1973).

8) Miyata, T. and F. Nakashio: Kagaku Kögaku, 37, 607 (1973).

9) Tanaka, M. and E. O'Shima, Kagaku Kogaku Ronbunshu, 9, 72 (1983). 